\title{
Study on anti-tumor activity of itraconazole in colon cancer
}

\author{
Xiaoqi $\mathrm{Li}^{1}$, Jingbo Shan ${ }^{1}$, Lijun $\operatorname{Jin}^{1}$, Xinyu $\mathrm{Li}^{1}, \mathrm{Sa}_{\text {Zhou }}{ }^{1, \mathrm{a}}$ and Wenjian $\mathrm{Ma}^{1, \mathrm{a}}$ \\ ${ }^{1}$ Key Laboratory of Industrial Microbiology, Ministry of Education and Tianjin City, College of Biotechnology, Tianjin University of \\ Science and Technology
}

\begin{abstract}
Itraconazole is a common antifungal drug, which inhibiting lanosterol 14 alpha-demethylase, interfering with lanosterol convert to ergosterol or cholesterol, thereby affecting the synthesis of fungal cell membranes. In recent years, it has been found that it has anti-cancer effect and is expected to be used to treat a variety of human cancers. Colon cancer is a cancer disease that affects the health of contemporary people and its incidence tends to be younger. In this paper, the effects of itraconazole on proliferation and migration of Caco2 and HT-29 of two kinds of colon cancer cells were detected by MTT, scratch experiment and western blot. The data showed that itraconazole concomitant with increased drug concentration caused cell morphology of colon cancer significantly shrink. MTT experiment showed that it could reduce the survival rate of colon cells and western blot data indicated that it could down-regulation PCNA and MYL9 which are marker genes of proliferation and migration. Furthermore, the cell migration is reduced.
\end{abstract}

\section{Introduction}

Itraconazole is a common antifungal agent that has been demonstrated reversal effects on P-glycoproteinmediated chemoresistance and the suppression of Akt/ mechanistic target of rapamycin (mTOR), Hedgehog $(\mathrm{Hh})$ and the WNT/ $\beta$-catenin pathway in various cancer cells[1]. It is a triazole antifungal drug, which has been used in clinical for more than 30 years. It has good pharmacokinetic characteristics, fast oral absorption, high tissue affinity, long half-life and lasting effect. Itraconazole's antibacterial effect is to inhibit lanosterol $14 \alpha$-demethylase, thus interfering with lanosterol's conversion to ergosterol or cholesterol and affecting the synthesis of fungal cell membrane. Survival benefit in cancer patients treated with itraconazole has been proposed in non-small cell lung, ovarian, triplenegative breast, pancreatic and biliary tract cancers[2]. However, the specific mechanism of anti-cancer is still unclear.

Colon cancer is a significant health problem and the leading cause of cancer mortality worldwide[3]. And its mortality rate is second only to lung cancer and liver cancer. Surgery seems to be the most effective therapeutic approach[4]. However, most patient condition had developed to the middle or late stage when they were diagnosed, and the recurrence rate was relatively high[5]. At present, the incidence of colon cancer is increasing year by year[6]. In order to cure colon cancer, the discovery of new drug functions has become a research hotspot, such as itraconazole. Especially in recent years, the study of intestinal flora and metabolites of intestinal flora, it has found that intestinal flora may participate in the formation of colon cancer. In addition, the origin of colon cancer is related to many external factors, such as high fat and low cellulose diet, living environment, water pollution, etc.

In this paper, two kinds of colon cancer cells Caco 2 and HT-29 are used as models. Itraconazole was found that it had anti-cancer effect, and the molecular mechanism of itraconazole in inhibiting cancer function was further explored. Our experiment indicated that cell morphology of Caco2 and HT-29 changes significantly and cell survival rate is decreasing with the itraconazole concentration increasing. In addition, our study show that itraconazole could inhibit the migration of colon cancer cells.

\section{Materials and Methods}

\subsection{Cells, reagents and instruments}

Colon cancer cells Caco2 and HT-29 have been stored in our laboratory for a long time. PCNA and MYL9 of primary antibody were purchased from abcam, GAPDH primary antibody and Santa Cruz. Goat anti-mouse fluorescent secondary antibody, Li-cor Company; Penicillin, streptomycin, pancreatin and MTT were all purchased from Beijing solable technology co, ltd. The Odyssey infrared laser imaging system used is li-cor Biosciences Company.

\subsection{Cell culture}

Colon cancer HT-29 uses D-MEM/F-12 medium culture containing $10 \%$ fetal bovine serum, $100 \mathrm{U} / \mathrm{mL}$ penicillin

a Corresponding author:ma_wj@tust.edu.cn, zhousa@tust.edu.cn 
and $0.1 \mathrm{mg} / \mathrm{mL}$ streptomycin. Caco2 was monolayer culture using DMEM-High Glucose of $15 \%$ AGX serum in a $37^{\circ} \mathrm{C}$ constant temperature incubator with saturated steam and $5 \% \mathrm{CO}_{2}$.

\subsection{MTT detection vitality and proliferation}

The cells were seeded into 96-well plates at a density of $5 \cdot 10^{3}$ cells per well, and after $12 \mathrm{~h}$, they were replaced with serum-free medium and synchronized (24 h). On the next day, cells were treated with different concentrations of itraconazole solution (final concentration $0,2,5,10,20,40,80,100 \mu \mathrm{M}$ ), placed in the incubator for $24 \mathrm{~h}$, and $10 \mu \mathrm{L}$ of MTT solution was added to each well $\left(5 \mathrm{mg} \cdot \mathrm{mL}^{-1}\right)$, after reacting for $4 \mathrm{~h}$ at $37^{\circ} \mathrm{C}$ in the dark, discard the medium and add to $100 \mu \mathrm{L}$ DMSO. The formed formazan crystals were measured for absorbance at a wavelength of $490 \mathrm{~nm}$ in a microplate reader to calculate relative cell viability. The formula is: Cell viability $(\%)=[\mathrm{OD}$ (treated)-OD (Blank)]/[OD (untreated)-OD (Blank)]×100.

\subsection{Western Blot}

Colon cancer cells were treated with Itraconazole and then extracted with protein lysate, and the obtained total cell proteins were separated by SDS-PAGE gel electrophoresis, transferred to $\mathrm{NC}$ membrane, sealed with primary antibody overnight at $4^{\circ} \mathrm{C}$, washed with PBS for three times( $5 \mathrm{~min}$ each time), incubated with secondary antibody in dark for $2 \mathrm{~h}$, then washed with PBS for three times (5min each time), and imaged NC membrane with Odyssey fluorescence imaging system.

\section{Results}

\subsection{Itraconazole changes the morphology of colon cancer cells.}

Itraconazole with concentrations of 0,5 and $10 \mu \mathrm{M}$ was prepared to treat $\mathrm{Caco} 2$ and HT-29 cells for 24 hours respectively. Compared with the control group, the cell morphology of the drug-treated group showed obvious shrinkage under the optical microscope. Apparently, slow cell proliferation was found and the number decreased. The cell morphology was shown in Fig. 1.

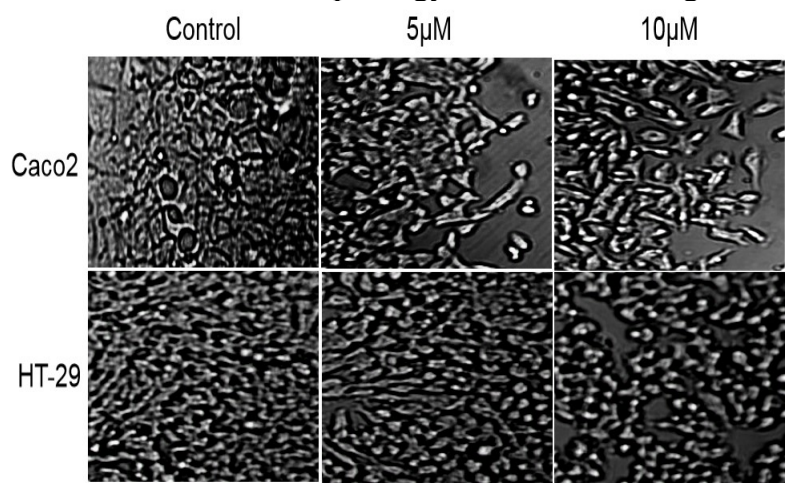

Figure 1. The morphology of colon cancer cells was observedafter $24 \mathrm{~h}$ treatment with Itraconazole.

\subsection{Effect of itraconazole on survival of colon cancer cells.}

Caco2 and HT-29 cells were treated with itraconazole at concentrations of $0,2,5,10,20,40,80$ and $100 \mu \mathrm{M}$ respectively. After 24 hours, the effect on the proliferation of the two cells is shown in Fig. 2. The results showed that itraconazole had obvious inhibitory effect on the proliferation of colon cancer cells at the concentration of $80 \mu \mathrm{M}$, which was concentration dependent and the cell survival rate decreased.

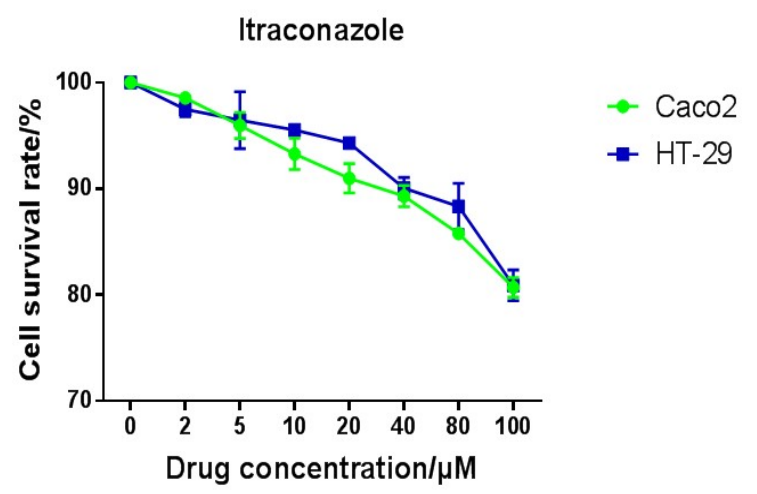

Figure 2. MTT assay for survival rate of colon cancer cells.

\subsection{Effect of itraconazole on PCNA protein level of colon cancer proliferation gene marker.}

In order to further determine the effect of Itraconazole on the proliferation of tumor cells. Caco2 and HT-29 cells were treated with Itraconazole for $24 \mathrm{~h}$ and then extracted with protein lysate for western blot. The results were shown in Fig. $3 a$ and $3 b$, the expression of proliferation marker PCNA was down-regulated, which indicated Itraconazole can further inhibit the proliferation of $\mathrm{Caco} 2$ and HT-29 cells.

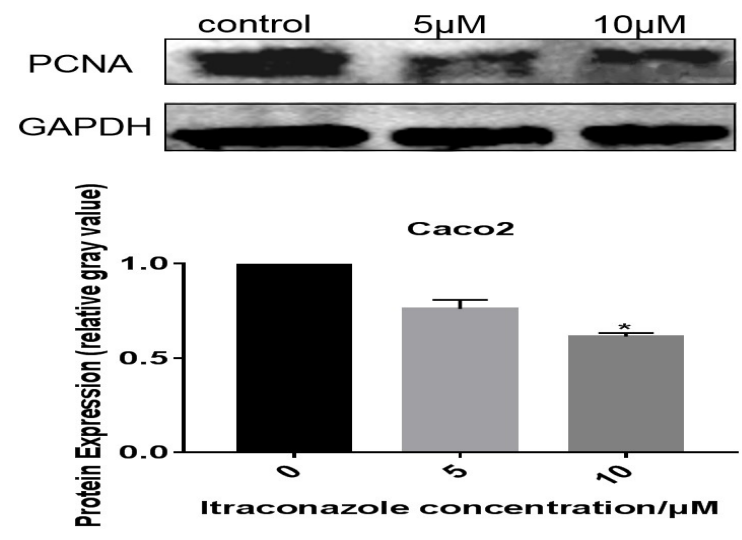

Figure 3a. The expression of Marker PCNA in Caco2. 

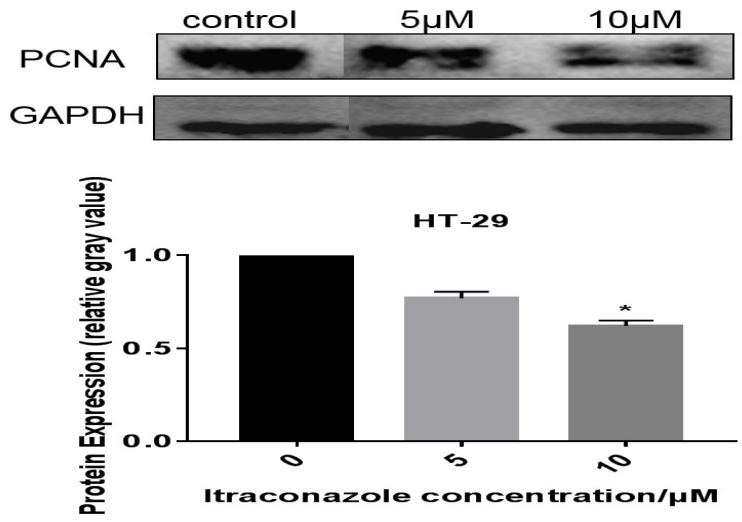

Figure 3b. The expression of Marker PCNA in HT-29.

\subsection{Scratch test to detect the effect of itraconazole on migration ability of colon cancer cells.}

Tumor migration refers to the characteristic that malignant tumor cells continue to grow from the primary site to other sites via lymphatic channels, blood vessels or body cavities. This is the most important biological behavior of malignant tumor and the main reason why it is difficult to cure and kill. As is shown in Fig. 4a and $4 \mathrm{~b}$, it is intuitively shown that the ability of cells to heal scratches greatly decreases with the increase of time and drug concentration.

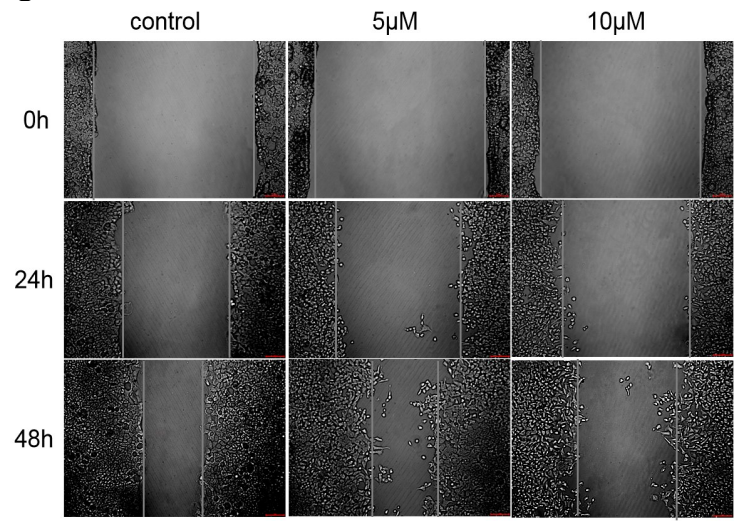

Figure 4a. The scratch assay assayed the effect of Itraconazole on $\mathrm{Caco} 2$ cell migration.

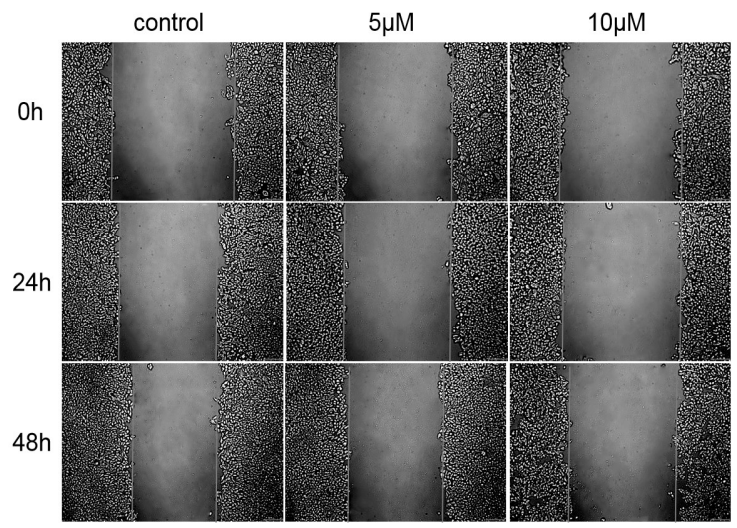

Figure $4 \mathbf{b}$. The scratch assay assayed the effect of Itraconazole on HT-29 cell migration.

\subsection{Effect of itraconazole on MYL9 protein level of colon cancer migration gene marker.}

As is shown in Fig. $5 \mathrm{a}$ and $5 \mathrm{~b}$, the results obtained by western blot after different concentrations of itraconazole which showed that the migration marker gene MYL9 was down-regulated at the protein level by concentration-dependent manner. The same trend as the scratch test results in Fig. $4 \mathrm{a}$ and $4 \mathrm{~b}$ shows that itraconazole has an inhibitory effect on the migration of colon cancer cells.

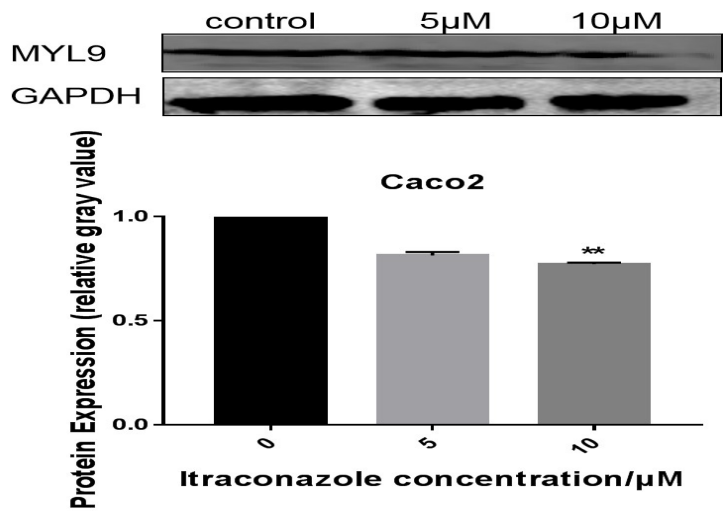

Figure 5a. The expression of Marker MYL9 in Caco2.

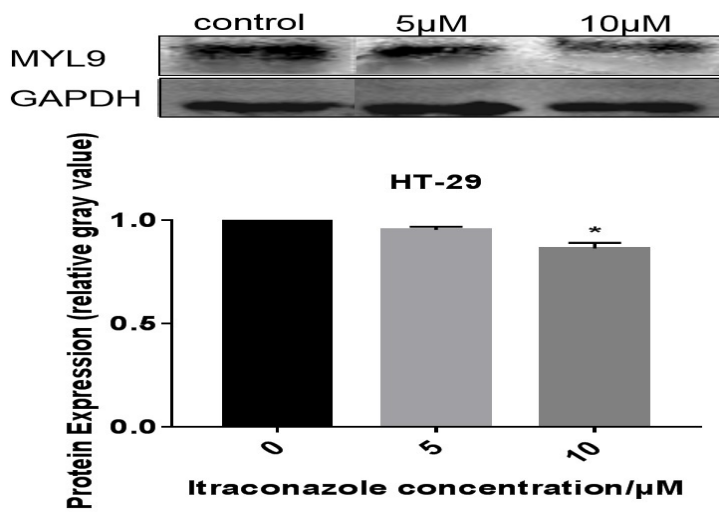

Figure 5b. The expression of Marker MYL9 in HT-29.

\section{Conclusions}

Colon cancer has a high incidence and mortality rate, and surgery is the common method for clinical treatment of colon cancer currently[7]. Itraconazole exerts its antifungal mechanism by acting on SMO protein [8] in Hedgehog signaling pathway, thus inhibiting transcription and translation of downstream Gli1 and other genes. At present, Itraconazole's second-stage clinical trials for the treatment of non-small cell lung cancer, basal cell cancer and prostate cancer are already in progress $[9,10,11,12]$. Invasion and metastasis are related to enhancement of tumor cell invasiveness and reduction of adhesion ability, angiogenesis, degradation of extracellular matrix, interstitial remodeling, etc. [13], which is the most common reason for failure in treatment of malignant tumors. In this paper, itraconazole was used to intervene colon cancer cells Caco2 and HT-29 to study the anti-tumor effect and 
molecular mechanism at the cellular level. The conclusions are as follows: Itraconazole can significantly change the morphology of colon cancer cells, reduce the cell survival rate, and inhibit cell migration. In addition, the proliferation and migration marker genes PCNA and MYL9 were down-regulated. That is, itraconazole can inhibit proliferation and migration of colon cancer cells. Itraconazole alone may have great limitations in treating tumor diseases, but its anti-tumor effect may be further strengthened by selectively blocking Hedgehog, Wnt or $\mathrm{PI} 3 \mathrm{~K} / \mathrm{mTOR}$ signal pathway in cooperation with other drugs.

\section{Conflict of Interest}

We have no conflicts of interest to disclose.

\section{References}

1. T. Ueda, H. Tsubamoto, K. Inoue, K. Sakata, H. Shibahara, T. Sonoda, Itraconazole Modulates Hedgehog, WNT/ $\beta$ - catenin, as well as Akt Signalling, and Inhibits Proliferation of Cervical Cancer Cells. Anticancer Res 7, 3521-3526(2017).

2. P. Pantziarka, V. Sukhatme, G.Bouche, L. Meheus, VP. Sukhatme, Repurposing Drugs in Oncology (ReDO)-itraconazole as an anti-cancer agent. Ecancermedicalscience 9, 521(2015).

3. S. Bahmani, N. Azarpira, E. Moazamian, Anti-colon cancer activity of Bifidobacterium metabolites on colon cancer cell line SW742. Turk J Gastroenterol 9, 835-842(2019).

4. X. Liang, M. Luo, XW. Wei, et al., A folate receptor-targeted lipoplex delivering interleukin-15 gene for colon cancer immunotherapy. Oncotarget 32, 52207-52217(2016).

5. K. Ogata, H. Takamori, N. Umezaki, et al., Gastrointestinal perforation during regorafenib administration in a case with hepatic metastases of colon cancer. J Chemother 5, 314-316(2017).

6. F. Agüero, C. Murta-Nascimento, M. Gallén, et al., Colorectal cancer survival: results from a hospitalbased cancer registry. Rev Esp Enferm Dig 11, 572577(2012).

7. Y. Tashiro, M. Murakami, K. Otsuka, et al., Intrathoracic Hernia after Total Gastrectomy. Case Rep Gastroenterol 1, 1-6(2016).

8. J. Kim, BT. Aftab, JY. Tang, et al., Itraconazole and arsenic trioxide inhibit Hedgehog pathway activation and tumor growth associated with acquired resistance to smoothened antagonists. Cancer Cell 1, 23-34(2013).

9. CM. Rudin, JR. Brahmer, RA. Juergens, et al., Phase 2 Study of Pemetrexed and Itraconazole as Second-Line Therapy for Metastatic Non-Squamous Non-Small Cell Lung Cancer. J Thorac Oncol 5, 619(2013).
10. DJ. Kim, J. Kim, K. Spaunhurst, et al., Open-label, exploratory phase II trial of oral itraconazole for the treatment of basal cell carcinoma. J Clin Oncol 8, 745(2014).

11. P. Pantziarka, V. Sukhatme, G. Bouche, L. Meheus, VP. Sukhatme, Repurposing Drugs in Oncology (ReDO)-itraconazole as an anti-cancer agent. Ecancermedicalscience 1, 485(2014).

12. RE. Kast, G. Karpel-Massler, ME. Halatsch, CUSP9* treatment protocol for recurrent glioblastoma: aprepitant, artesunate, auranofin, captopril, celecoxib, disulfiram, itraconazole, ritonavir, sertraline augmenting continuous low dose temozolomid. Oncotarget 18, 8052-8082(2014).

13. X. Yio, M. Diamond, L. Werther, et al., Trefoil factor family-1 mutations enhance gastric cancer cell invasion through distinct signaling pathways. Gastroenterology 6, 1696-1706(2006). 